\title{
AJUSTE DE MODELOS DE VOLUMEN Y FUNCIONES AHUSAMIENTO PARA Pinus teocote EN BOSQUES DE PINO DE LA SIERRA MADRE ORIENTAL
}

\author{
JUAN TAPIA $\left({ }^{*}\right)$ y JOSÉ NÁVAR $\left({ }^{* *}\right)$
}

(*) Estudiante de Maestría en Ciencias Forestales.

(**) Profesor investigador.

Facultad de Ciencias Forestales, UANL. Km. 145 Carretera Nacional Linares, N. L. 67700 México. Email: jnavar@ccr.dsi.uanl.mx

\section{RESUMEN}

El presente trabajo tuvo como objetivos ajustar ocho modelos de volumen y cinco funciones de ahusamiento y comparar su bondad de ajuste, para la especie Pinus teocote de los predios "El Rancho el 18" y "Las Anacuas", situados en la Sierra Madre Oriental. Las mediciones de diámetro y altura a diferentes secciones del fuste se realizaron en árboles derribados en operaciones de manejo forestal. Para el ajuste de las funciones de volumen y ahusamiento se utilizaron 1.281 pares de datos diámetro - altura correspondientes a 110 árboles y para la bondad de ajuste de 424 pares de datos de 20 árboles seleccionados al azar. Los modelos de volumen fueron ajustados con los datos de 110 árboles y su bondad probada con los 20 árboles de la muestra de los datos. Los modelos y funciones fueron ajustadas, por medio de la técnica de regresión lineal, intrínsecamente lineal, polinomial, múltiple. Las pruebas de bondad de ajuste consistieron en comparar los estadísticos; coeficiente de determinación, el error estándar y el sesgo, incluyendo la distribución, normalidad y variancia común de los errores. Los resultados mostraron que los modelos que predicen mejor el volumen y el perfil diamétrico fueron el de Schumacher y Hall y el de Newnham, respectivamente. Los modelos de volumen predicen mejor el volumen que las funciones de ahusamiento para 20 árboles utilizados en las pruebas de bondad de ajuste. Los modelos de volumen desarrollados para la misma especie en otros rodales de la Sierra Madre Oriental y Occidental no mostraron diferencias estadísticamente significativas con los modelos desarrollados en este reporte.

Palabras Clave: Pinus teocote, Modelos de Volumen, Funciones de Ahusamiento, Sierra Madre Oriental. 


\begin{abstract}
This research had as objectives to fit eight volume equations and five taper functions and to compare the goodness of fit of these models to observed volumes and stem profiles of the species Pinus teocote of the private property "El Rancho el 18" and "Las Anacuas", located in the eastern Sierra Madre. Diameter at different stem sections were measured from fallen trees during harvesting operations. To fit the taper and volume functions, 1.281 pairs of data of 110 trees were used. To test the goodness of fit, 424 pairs of data from 20 trees randomly selected were used. The model parameters were estimated by least square techniques in linear, non-linear, polynomial, and multiple regression techniques. Tests of goodness of fit consisted in comparing the statistics: coefficient of determination, standard error and skewness, including the error distribution, randomness and common variance. The results showed that the models of Schumacher and Hall and Newnhamm described better stem volumes and stem profiles of the studied species, respectively. The volume models described better volume of the 20 randomly selected trees than the taper functions. Volume models developed for the same species in other forest stands of the eastern and western Sierra Madre did not deviated from the model developed in this study.
\end{abstract}

Keywords: Pinus teocote, Volume Models, Taper Functions, Eastern Sierra Madre. 


\section{INTRODUCCIÓN}

La determinación de volumen y la distribución de productos forestales por medio de ecuaciones de volumen $\mathrm{y}$ funciones de ahusamiento, es una de las principales herramientas utilizadas por el manejador de bosques en la elaboración de planes de manejo e inventarios forestales. Estas tecnologías matemáticas se han utilizado desde hace varias décadas, mientras que en México estas herramientas han tardado en incorporarse al manejo forestal al nivel de la especie comercial.

Las ecuaciones de volumen que se han ajustado son bastantes, Jiménez $(1988,1990)$ reporta la utilización de 50 de éstas, para la elaboración de tablas de volumen para Pinus pseudostrobus y Pinus teocote en una fracción de la Sierra Madre Oriental y la aplicación de un modelo matemático para la elaboración de tablas y tarifas de volumen con Pinus pseudostrobus. En Durango, Márquez et al (1995), ajusta ecuaciones de volumen para el género Pinus y Herrera (1995) elabora tablas de volumen y tarifas para Pinus hartwgii en el Cerro Potosí, Galeana, Nuevo León. También se han ajustado modelos de volumen para Pinus cembroides en la Sierra de Arteaga, Coahuila (Navarro et al, 1997), Pinus rudis en el Cerro Potosí (Baca, et al 1997), y más recientemente para Pinus teocote en la Sierra Madre Occidental (Contreras, 1997) del estado de Durango.

Las funciones de ahusamiento también se han ajustado a varias especies de coníferas del país. Niembro (1992) utiliza tres funciones para determinar la forma externa de la especie de Pinus hartwgii del Noreste del estado de Nuevo León. Torres et al., (1993) prueba varios modelos para ocho especies forestales del estado de México. Jiménez (1994) también utiliza tres funciones para determinar la forma externa de Pinus hartwgii en el Noreste de México. Rentería (1995) estima el volumen comercial de Pinus cooperi mediante modelos de ahusamiento en el estado de Durango. De los Santos (1995) ajusta el modelo compatible de volumen-ahusamiento de Bailey para Pinus cooperi y Pinus patula y Návar et al., (1997), para Pinus hartwgii. Zepeda et al., (1997), prueba varias funciones para las especies de Pinus arizonica, Pinus durangensis y Pinus engelmani en la Sierra Madre Occidental del estado de Chihuahua.

Para la especie de Pinus teocote se han probado modelos de volumen para la Sierra Madre Oriental (Jiménez, 1988) y en Durango para la Sierra Madre Occidental (Contreras, 1997). Esta especie por poseer una plasticidad ecológica muy amplia y por su importancia económica merece una mayor atención local. Para contar con información confiable para desarrollar los planes de manejo sustentables de los bosques del Noreste de México.

Los objetivos de este trabajo fueron: 1) ajustar y probar la bondad de ajuste de ocho modelos de volumen y cinco funciones de ahusamiento y, 2) observar las diferencias con Pinus teocote de otros rodales de la Sierra Madre Oriental y la Sierra Madre Occidental. 


\section{MATERIALES Y MÉTODOS}

\section{Descripción General del Área de Estudio}

Localización

El estudio se realizó en el predio particular "El 18" y "Las Anacuas", los cuales se localizan en el sistema montañoso llamado Sierra Madre Oriental y se encuentra entre las coordenadas $99^{\circ} 43^{\prime}$ y $99^{\circ} 49^{\prime}$ de longitud Oeste y $24^{\circ} 41^{\prime}$ y $24^{\circ} 50^{\prime}$ de latitud Norte. Los predios se encuentran a 22,5 kilómetros de Linares. Las alturas sobre el nivel del mar fluctúan entre 700 y 2.200 metros. Las principales elevaciones de la zona son: "Sierra El Gabacho" con 2.200 msnm, con topografía muy accidentada, pendientes que fluctúan entre el 35 y $70 \%$ y algunas pequeñas áreas planas de uso pecuario.

Clima

De acuerdo al sistema de clasificación de Köppen modificado por Enriqueta García (1985), el predio está comprendido dentro del grupo de climas templados C, sub-grupo de climas semicálidos (A) C (Wo) y tipos semicálidos sub-húmedos con lluvias en verano con un porciento de lluvia invernal entre 5 y $10,2 \mathrm{~mm}$. La precipitación media anual es de $800-1.000 \mathrm{~mm}$ y una frecuencia alta de granizadas. La temperatura media anual es de $20-22^{\circ} \mathrm{C}$.

Geología

La geología del predio consiste en lutitas y calizas del cretásico superior (Ks) y cretásico inferior (Ki). Las rocas son del tipo sedimentario y vulcanoso sedimentario. Los tipos de suelos encontrados en el predio son litosol y rendzina, como suelo secundario encontrado en menor porcentaje el regosol calcárico de textura media.

Estado Forestal

Por su ubicación geográfica, esta zona presenta diversas condiciones de vegetación que va desde el matorral sub-montano, chaparral, masas puras de encino y bosque de pino encino. La vegetación arbolada aprovechable está compuesta por masas arboladas de bosque de pino encino y masas puras de encino distribuidas en el predio, las especies aprovechables son las siguientes: Pinus teocote, Pinus pseudostrobus, Quercus cambyi, Quercus prinopsis y Quercus cupreata. 


\section{Descripción de la Especie}

Este pino tiene una amplia zona de distribución y presenta, por lo mismo, muchas variaciones en sus conos, sus hojas y formas de fustes. Algunas formas del Sur tienen semejanza con el Pinus lawsoni y algunas del Norte con el Pinus arizonica. Se le conoce comúnmente como pino chino u ocote y es un árbol por lo común de 10 a 20 metros, pero varía entre los 8 y 25 ; de copa redondeada e irregular, ramas desigualmente distribuidas con follaje denso y erguido; corteza de color grisáceo por fuera y algo naranja o amarillento por dentro, delgada al principio y después áspera y rugosa dividida en grandes placas longitudinales. El tronco emite ocasionalmente retoños, asemejándose en esto al Pinus leiophylla. Hojas en grupos de tres, por rareza dos o cuatro en algunos fascículos, por lo general de 10 a $15 \mathrm{~cm}$, fuertes y tiesas, anchas y hasta cerca de 2 milímetros, por excepción delgada de bordes aserrados, su color es verde brillante, comúnmente con tinte amarillento, con estomas en las tres caras. Las vainas son persistentes de 5 a 8 milímetros en los fascículos adultos, de 10 a 15 en los jóvenes, escamosas y de color castaño obscuro. Conos ovoides u ovoide cónicos, rara vez subcilíndricos de 4 a $6,5 \mathrm{~cm}$, a veces 7 , simétricos o casi simétricos, por lo común reflejados en cortos pedúnculos de 5 a $8 \mathrm{~mm}$, pero a veces de 10 a 12 y en ocasiones subsésiles o sésiles. Por lo general son pronto caedizos de color moreno algo lustroso a veces con tinte ocre o rojizo, se encuentran por pares, pero a veces solitarios o en grupos de tres o cuatro. La madera es fuerte y de buena calidad y se usa para construcciones, como combustible y productor de trementina (Martínez, 1948). Su distribución es muy amplia y generalmente se encuentra en alturas que varían de 1.400 a $3.000 \mathrm{msnm}$ desde $15^{\circ} 00^{\prime}$ hasta $25^{\circ} 10^{\prime}$ de altitud Norte y $91^{\circ} 30^{\prime}$ a $108^{\circ} 00^{\prime}$ de longitud Oeste. En México desde el Sur de Chihuahua a lo largo de la Sierra Madre Occidental hasta Chiapas y en la Sierra Madre Oriental desde el Sur de Coahuila hasta Hidalgo y Puebla.

\section{Metodología}

Para cumplir los objetivos de esta investigación se midieron los diámetros a diferentes secciones de la altura de árboles derribados en operaciones de extracción por sus propietarios y personal técnico de la Facultad de Ciencias Forestales. El número de árboles muestreados fue de 130 porque Tapia y Návar (1997) estimaron que un mínimo de 100 a 140 árboles sería necesario para el ajuste de los modelos de volumen y ecuaciones de ahusamiento. Esta variable fue medida a la base del fuste, a 0,50 m, a 1,30 $\mathrm{m}$, a 1,50 y desde aquí hasta la parte distal a cada $0,50 \mathrm{~m}$. Otras variables medidas fueron: edad, altura del tocón, grosor de albura, grosor de corteza y altura total. El volumen total se calculó a través de la formula descrita por Smalian en 1824. 
Modelos de Volumen Ajustados

Los modelos de volumen ajustados fueron los siguientes:

1. Ecuación de Spurr (1952).

$V=\beta_{0}+\beta_{1}^{*} D^{2} H$

2. Ecuación de Spurr en forma polinomial de segundo grado.

$V=\beta_{0}+\beta_{1}^{*} D^{2} H+\beta_{2}^{*}\left(D^{2} H\right)^{2}$

3. Ecuación de Spurr en forma polinomial de tercer grado.

$V=\beta_{0}+\beta_{1}^{*} D^{2} H+\beta_{2}^{*}\left(D^{2} H\right)^{2}+\beta_{3}^{*}\left(D^{2} H\right)^{3}$

4. Ecuación de Naslund.

$V=\beta_{0}+\beta_{1}^{*} H+\beta_{2}^{*} D H+\beta_{3}^{*} D^{2} H$

5. Ecuación Australiana.

$V=\beta_{0}+\beta_{1}^{*} D^{2}+\beta_{2}^{*} H+\beta_{3}^{*} D^{2} H$

6. Ecuación múltiple de tres parámetros.

$V=\beta_{0}+\beta_{1}^{*} D^{2} H+\beta_{2}^{*} H+\beta_{3}^{*} D H^{2}$

7. Ecuación de Schumacher y Hall (1933).

$L V(V)=L N\left(\beta_{0}\right)+\beta_{1}^{*} L N(D)+\beta_{2}^{*} L N(H)$

8. Ecuación de Spurr logarítmica.

$L V(V)=L N\left(\beta_{0}\right)+\beta_{1}^{*} L N\left(D^{2} H\right)$

donde:

$V=$ volumen $\left(\mathrm{m}^{3}\right)$

$D=$ diámetro a la altura del pecho (dap), diámetro normal o a $1,3 \mathrm{~m}$

$H=$ altura total $(\mathrm{m})$

$L N=$ logaritmo natural

$\beta_{1}, \beta_{2}, \beta_{3}, \beta_{4}=$ parámetros estadísticos 
Los modelos para describir el perfil diamétrico de los árboles fueron los siguientes:

1. Amidon (1984).

$d=\beta_{0} \frac{D(H-h)}{H-1,3}+\beta_{1} \frac{\left(H^{2}-h^{2}\right)(h-1,3)}{H^{2}}$

2. Clutter (1980).

$d=\beta_{1} * D^{\beta_{2}} * H^{\beta_{3}}(H-h)^{\beta_{4}}$

3. Kozak (1988).

$\ln (d)=f\left(\ln \left(X_{k}\right), Z, \ln (Z), \exp ^{z}, \sqrt{Z,} \frac{D}{H}\right)$

4. Modificado de la forma variable presentado por Newnham (1992).

$$
\ln \left(\frac{d}{D}\right)=f\left(X, \ln (X), \frac{D}{H}, \frac{\frac{D}{H}}{\sqrt{h}}, \frac{H}{\sqrt{h}}\right)
$$

5. Rustagi y Loveless (1991).

$d=\left(\beta_{0}+\beta_{1} D\right)\left[\frac{H-h}{H_{a}}\right]^{\beta}$

donde:

$d=$ diámetro del fuste a la altura $\mathrm{h}(\mathrm{m})$

$h=$ Altura del fuste sobre el tocón (m)

$X=(\mathrm{H}-\mathrm{h}) /(\mathrm{H}-1,30 \mathrm{~m})$ (sin dimensiones)

$X_{k}=\left(1-\mathrm{Z}^{-1 / 2}\right) /\left(1-\mathrm{P}^{-1 / 2}\right)($ sin dimensiones $)$

$Z=\mathrm{h} / \mathrm{H}$ (sin dimensiones)

$H_{a}=\mathrm{H}-1,30 \mathrm{~m}$

Procedimiento Estadístico

En el ajuste de las ecuaciones de volumen y funciones de ahusamiento se utilizaron 110 y 2.181 datos de diámetro y altura, con los cuales se estimaron los parámetros estadísticos de los ocho modelos de volumen y de las cinco ecuaciones de ahusamiento. Para el ajuste se utilizó la técnica de cuadrados mínimos en regresión lineal, intrínsecamente lineal, múltiple, polinomial y no lineal. Para las ecuaciones que necesitaron transformaciones, los parámetros estadísticos se calcularon con las 
transformaciones necesarias, pero el coeficiente de determinación $\left(\mathrm{r}^{2}\right)$ y el error estándar (EEE) se estimaron con los volúmenes regresados a sus dimensiones originales.

La prueba de bondad de ajuste se realizó para los modelos de volumen y ahusamiento, con los datos de diámetro-altura del $15 \%$ de la muestra correspondientes a 20 árboles, que fueron seleccionados al azar de los 130 medidos, a través del sesgo promedio, $r^{2}$ y el EEE. Estos estadísticos se estimaron como sigue:

Sesgo Pr omedio $=\frac{\sum_{\mathrm{i}=1}^{\mathrm{n}}\left(\mathrm{Y}_{\mathrm{i}}-\tilde{\mathrm{Y}}_{\mathrm{i}}\right)}{\mathrm{n}}$

$$
r=1-\frac{\sum_{i=1}^{n}\left(Y_{i}-\tilde{Y}_{i}\right)^{2}}{\sum_{i=1}^{u}\left(Y_{i}-\bar{Y}\right)^{2}}
$$

$E E E=\left[\frac{\sum_{i=1}^{n}\left(Y_{i}-\tilde{Y}_{i}\right)^{2}}{n-p}\right]^{1 / 2}$

donde:

$Y i=$ valor observado o la variable dependiente

$\bar{Y}=$ promedio de los dato observados

$\tilde{Y}=$ valor predicho.

Finalmente se graficaron los errores para observar su distribución, aleatoriedad y variancia común y tener una mayor certidumbre en la selección de los modelos. La determinación del mejor modelo resultó de la ponderación de los diferentes parámetros, los cuales se califican por orden de importancia y fueron los siguientes: $r^{2}$, EEE, sesgo promedio, distribución de los errores en función de la altura y el diámetro del 85 y $15 \%$, la normalidad de los errores también para el 85 y $15 \%$, el número de los coeficientes de las regresiones así como el valor de su probabilidad, y finalmente la observación de las tablas de volumen resultantes. Para las funciones de ahusamiento, se utilizó principalmente el $\mathrm{r}^{2}$, EEE y el sesgo, otros parámetros como el número de variables, gráficas de la distribución del error en función de la altura para el 85 y $15 \%$, histogramas para determinar la normalidad de los errores y finalmente la graficación de los datos observados contra los predichos para diferentes categorías diamétricas. 
Para comparar los volúmenes observados y predichos por las funciones de volumen y de ahusamiento, el volumen calculado por sección y árbol fue determinado por la fórmula de Smalian para ser consistentes en la comparación.

\section{Características de los Árboles Muestra}

Los estadísticos de los parámetros dasométricos de los árboles, separados en $85 \%$ y $15 \%$ respectivamente se presentan en el Cuadro 1 . Donde se observa que no existen diferencias significativas en sus estadísticas principales.

Cuadro 1.

ESTADÍSTICAS DE LOS DATOS DE LOS ÁRBOLES DE PINUS TEOCOTE PARA EL $85 \%$ Y $15 \%$, RESPECTIVAMENTE.

\begin{tabular}{|l|c|c|c|c|c|c|}
\hline \multicolumn{5}{|c|}{$85 \%$} & \multicolumn{3}{c|}{$15 \%$} \\
\hline ESTADÍsTICOS & $\begin{array}{c}\text { DIÁMETRO } \\
(\mathrm{m})\end{array}$ & $\begin{array}{c}\text { ALTURA } \\
(\mathrm{m})\end{array}$ & $\begin{array}{c}\text { EDAD } \\
(\mathrm{años})\end{array}$ & $\begin{array}{c}\text { DIÁMETRO } \\
(\mathrm{m})\end{array}$ & $\begin{array}{c}\text { ALTURA } \\
(\mathrm{m})\end{array}$ & $\begin{array}{c}\text { EDAD } \\
(\mathrm{años})\end{array}$ \\
\hline Promedio & 0,248 & 17,053 & 54,136 & 0,268 & 18,435 & 56,200 \\
\hline Des. Estándar & 0,068 & 2,720 & 13,019 & 0,061 & 2,570 & 9,774 \\
\hline Sesgo & 0,451 & $-0,152$ & $-0,034$ & $-0,156$ & $-0,110$ & $-0,503$ \\
\hline
\end{tabular}

\section{RESULTADOS Y DISCUSIÓN}

Los parámetros generados para cada uno de los modelos de volumen se presentan en el Cuadro 2.

\section{Cuadro 2.}

\section{PARÁMETROS DE LOS MODELOS DE VOLUMEN PARA LA ESPECIE Pinus teocote DE} UNA FRACCIÓN DE LA SIERRA MADRE ORIENTAL EN EL ESTADO DE NUEVO LEÓN.

\begin{tabular}{|c|l|}
\hline \multicolumn{2}{|c|}{ MODELOS } \\
\hline 1 & $\mathrm{~V}=0,033415+0.370801^{*} \mathrm{D}^{2} \mathrm{H}$ \\
\hline 2 & $\mathrm{~V}=-0,019597+0.464355 * \mathrm{D}^{2} \mathrm{H}-0,029014^{*}\left(\mathrm{D}^{2} \mathrm{H}\right)^{2}$ \\
\hline 3 & $\mathrm{~V}=0,007783+0.390594^{*} \mathrm{D}^{2} \mathrm{H}+0,020041^{*}\left(\mathrm{D}^{2} \mathrm{H}\right)^{2}-0,008867 *\left(\mathrm{D}^{2} \mathrm{H}\right)^{3}$ \\
\hline 4 & $\mathrm{~V}=0,055580-0.019208^{*} \mathrm{H}+0,138061^{*} \mathrm{DH}+0,122902 * \mathrm{D}^{2} \mathrm{H}$ \\
\hline 5 & $\mathrm{~V}=-0,077589+1.542530 * \mathrm{D}^{2}+0,006597 * \mathrm{H}+0,283258 * \mathrm{D}^{2} \mathrm{H}$ \\
\hline 6 & $\mathrm{~V}=0,135616+0.302189 * \mathrm{D}^{2} \mathrm{H}-0,009802 * \mathrm{H}+0,001885 * \mathrm{DH} \mathrm{H}^{2}$ \\
\hline 7 & $\mathrm{LV}(\mathrm{V})=\mathrm{LN}(-0,600417)+2.008682 * \mathrm{LN}(\mathrm{D})+0,894068 * \mathrm{LN}(\mathrm{H})$ \\
\hline 8 & $\mathrm{LV}(\mathrm{V})=\mathrm{LN}(-0,912448)+0,984238^{*} \mathrm{LN}\left(\mathrm{D}^{2} \mathrm{H}\right)$ \\
\hline
\end{tabular}

De los modelos utilizados los que presentaron un mejor ajuste fueron: 2,3 y 4 con los $r^{2}$ más altos y los EEE. El $r^{2}$ y EEE entre los modelos no muestran diferencias notorias (Cuadro 3). En la prueba de bondad de ajuste los mejores modelos fueron 1,7 y 8, a 
diferencia de los observados en la prueba de ajuste discutida anteriormente. El menor sesgo lo presentan los modelos 2, 3, y 4 para el $85 \%$ y los modelos 1,7 , y 8, para el 15 $\%$. Estos números son consistentes para el 85 y el $15 \%$ de la información.

Los errores se distribuyeron más aleatoriamente y más normalmente en contra del diámetro y altura en los modelos del 1 al 6 y ligeramente dispersos en los modelos 7 y 8 . Las tablas de volumen generadas, las cuales son la herramienta práctica del resultado de estas investigaciones, para los modelos $2,3,4,5$ y 6 presentaron valores negativos y en ocasiones muy altos. Considerando la ponderación de los parámetros considerados, los estadísticos de ajuste y bondad de ajuste, características de los errores y las tablas de volumen generadas se concluye que el modelo 7, de Schumacher y Hall, resulta en el modelo mas adecuado para predecir los volúmenes fustales para la especie estudiada. Este modelo ha sido también recomendado por Contreras (1997) para estimar además volúmenes rollo total árbol, volumen fuste y ramas, volumen fuste y tocón, con y sin corteza para Pinus teocote. Este es también el modelo utilizado por El Inventario Nacional Forestal en sus actividades de evaluación de los recursos forestales de la Nación (SARH, 1994).

Cuadro 3.

ESTADÍSTICOS DEL COEFICIENTE DE REGRESIÓN, ERROR ESTÁNDAR Y NÚMERO DE COEFICIENTES PARA LOS OCHO MODELOS DE VOLUMEN AJUSTADOS A Pinus teocote EN UNA FRACCIÓN DE LA SIERRA MADRE ORIENTAL DEL ESTADO DE NUEVO LEÓN.

\begin{tabular}{|l|c|c|c|c|c|}
\hline Modelo Volumen Fustal & \multicolumn{2}{|c|}{$85 \%$ muestra } & \multicolumn{3}{c|}{$15 \%$ muestra } \\
\hline & $R^{2}$ & EEE & $\mathrm{N}^{\circ} \mathrm{de} \beta$ & $\mathrm{R}^{2}$ & EEE \\
\hline $1 \mathrm{~V}=\beta_{0}+\beta_{1} * \mathrm{D}^{2} \mathrm{H}$ & 0,9626 & 0,05680 & 2 & 0,9505 & 0,05037 \\
\hline $2 \mathrm{~V}=\beta_{0}+\beta_{1} \mathrm{D}^{2} \mathrm{H}+\beta_{2} *\left(\mathrm{D}^{2} \mathrm{H}\right)^{2}$ & 0,9682 & 0,05263 & 2 & 0,9651 & 0,05674 \\
\hline $3 \mathrm{~V}=\beta_{0}+\beta_{1} * \mathrm{D}^{2} \mathrm{H}+\beta_{2} *\left(\mathrm{D}^{2} \mathrm{H}\right)^{2}+\beta_{3} *\left(\mathrm{D}^{2} \mathrm{H}\right)^{3}$ & 0,9688 & 0,05236 & 1 & 0,9668 & 0,05699 \\
\hline $4 \mathrm{~V}=\beta_{0}+\beta_{1}{ }^{*} \mathrm{H}+\beta_{2} * \mathrm{DH}+\beta_{3} * \mathrm{D}^{2} \mathrm{H}$ & 0,9686 & 0,05248 & 3 & 0,9630 & 0,06022 \\
\hline $5 \mathrm{~V}=\beta_{0}+\beta_{1} * \mathrm{D}^{2}+\beta_{2} * \mathrm{H}+\beta_{3} * \mathrm{D}^{2} \mathrm{H}$ & 0,9635 & 0,05659 & 1 & 0,9685 & 0,05553 \\
\hline $6 \mathrm{~V}=\beta_{0}+\beta_{1} * \mathrm{D}^{2} \mathrm{H}+\beta_{2} * \mathrm{H}+\beta_{3} * \mathrm{DH}$ & 0,9626 & 0,05653 & 1 & 0,9701 & 0,05410 \\
\hline $7 \mathrm{LV}(\mathrm{V})=\mathrm{LN}\left(\beta_{0}\right)+\beta_{1}{ }^{*} \mathrm{LN}(\mathrm{D})+\beta_{2} * \mathrm{LN}(\mathrm{H})$ & 0,9585 & 0,06001 & 3 & 0,9720 & 0,05077 \\
\hline $8 \mathrm{LV}(\mathrm{V})=\mathrm{LN}\left(\beta_{0}\right)+\beta_{1} * \mathrm{LN}\left(\mathrm{D}^{2} \mathrm{H}\right)$ & 0,9443 & 0,06921 & 2 & 0,9245 & 0,08109 \\
\hline
\end{tabular}

$\mathbf{N}^{\circ} \beta=$ parámetros con valores de probabilidad aceptada.

\section{Funciones de Ahusamiento}

Las funciones que se ajustaron mejor a los perfiles fustales fueron: 1) Newnham y 2) Rustagi y Loveless porque presentaron los valores de $r^{2}$ más altos, los errores estándar más bajos y los sesgos menores (Cuadro 4). Las funciones que presentaron mejor bondad de ajuste fueron: 1) Newnham y 2) Amidon, las cuales presentaron valores de $r^{2}$ altos, valores del error estándar bajos y ambos con sesgos diferentes. Es decir, el modelo de Amidon subestima y el modelo de Newnham sobrestima el diámetro de los árboles. Este sesgo no posee un valor absoluto muy grande, y se encuentra entre 0,15 y $0,036 \mathrm{~cm}$ 
como promedio para los modelos, respectivamente (Cuadro 5). A este respecto, la ecuación de Rustagi y Loveless presentó un menor valor del sesgo, pero un valor menor del $\mathrm{r}^{2} \mathrm{y}$ un valor mayor del error estándar.

Cuadro 4.

ESTADÍSTICOS RESULTANTES DEL AJUSTE DE CINCO FUNCIONES DE AHUSAMIENTO PARA 110 ÁRBOLES DE LA ESPECIE Pinus teocote DE UNA FRACCIÓN DE LA SIERRA MADRE ORIENTAL DEL SUR DE NUEVO LEÓN.

\begin{tabular}{|l|c|c|c|c|}
\hline MODELOS & VARIABLES & SESGO PROM. & $\mathrm{R}^{2}$ & EEE \\
\hline Amidon & 02 & 0,00139969 & 0,942842 & 0,022720 \\
\hline Clutter & 04 & 0,00274428 & 0,939763 & 0,023329 \\
\hline Kozak & 11 & 0,00278938 & 0,937301 & 0,023180 \\
\hline Newnham & 16 & $-0,00099421$ & 0,973540 & 0,015505 \\
\hline Rus. y Lov. & 03 & 0,00000403 & 0,944593 & 0,022374 \\
\hline
\end{tabular}

Cuadro 5 .

ESTAdíSTICOS RESULTANTES DE LA PRUEBA DE BONDAD DE AJUSTE DE CINCO FUNCIONES DE AHUSAMIENTO A 20 ÁRBOLES DE LA ESPECIE Pinus teocote DE UNA FRACCIÓN DE LA SIERRA MADRE ORIENTAL DEL SUR DE NUEVO LEÓN.

\begin{tabular}{|l|c|c|c|c|}
\hline MODELOS & VARIABLES & SESGO PROM. & $\mathbf{R}^{2}$ & EEE \\
\hline Amidon & 02 & 0,00156957 & 0,976218 & 0,014756 \\
\hline Clutter & 04 & 0,00424889 & 0,942817 & 0,022865 \\
\hline Kozak & 11 & 0,00232996 & 0,944366 & 0,022345 \\
\hline Newnham & 16 & $-0,00036189$ & 0,978027 & 0,014391 \\
\hline Rus. y Lov. & 03 & 0,00096236 & 0,975831 & 0,014858 \\
\hline
\end{tabular}

Los modelos de Newnham y de Kozak predicen adecuadamente las curvas sigmoidales de los perfiles diamétricos, mientras que el resto de los modelos simulan curvas cuadráticas sencillas (Figura 1). Árboles con diámetros mayores que $40 \mathrm{~cm}$ presentan un ahusamiento mayor en la base del fuste y ninguno de los dos primeros modelos simula correctamente este comportamiento y generalmente subestiman el diámetro a la base del tocón. Los diámetros distales de los fustes predichos por el modelo de Newnham se aproximan al 0 absoluto pero no convergen a este valor y generalmente sobrestiman el diámetro en este punto. Los errores de todos los modelos se distribuyeron aleatoria y normalmente, con las máximas frecuencias en el 0 y mostraron variancia común.

El modelo de Newnham, consistente con las observaciones de Contreras (1997) para la misma especie pero en la Sierra Madre Occidental, se ajustó mejor a los perfiles diamétricos de la especie Pinus teocote de la Sierra Madre Oriental. A este respecto, no se han realizado otros trabajos para ajustar ahusamiento a esa especie en otros rodales del estado de Nuevo León. 


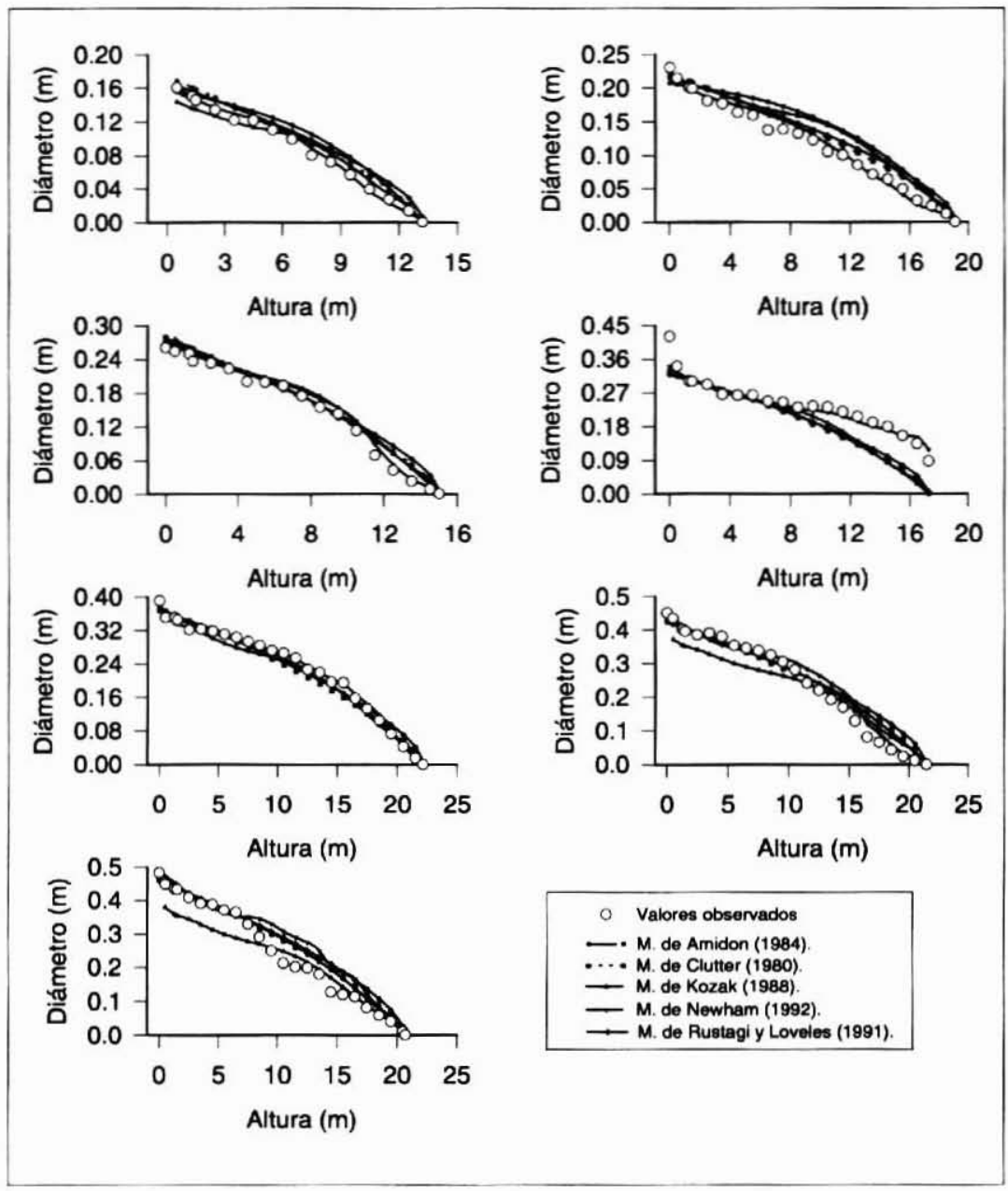

Figura 1. AJUSTE DE CINCO MODELOS DE AHUSAMIENTO A LOS PERFILES FUSTALES DE ÁRBOLES DE DIFERENTES CATEGORÍAS DIAMÉTRICAS DE Pinus teocote.

Los volúmenes fustales observados de los 20 árboles utilizados en la prueba de bondad de ajuste, y estimados por las ecuaciones de ahusamiento y por las ecuaciones de volumen se presentan en el Cuadro 6. 
Cuadro 6.

COMPARACIÓN DEL VOLUMEN FUSTAL DE 20 ÁRBOLES ESTIMADOS POR ECUACIONES DE VOLUMEN Y AHUSAMIENTO.

\begin{tabular}{|c|c|c|c|c|c|c|c|c|c|c|c|c|}
\hline $\mathrm{N}^{\circ}$ & $\mathrm{H}_{(\mathrm{mi})}$ & $D_{(\mathrm{m})}$ & vo & $\mathrm{VAl}$ & $\mathrm{VA2}$ & $\mathrm{VA}^{3}$ & VA4 & VAS & Ml & M6 & M7 & M8 \\
\hline 1 & 18,10 & 0.256 & 0,466 & 0,479 & 0.463 & 0,485 & 0,463 & 0,468 & 0,473 & 0,474 & 0,473 & 0,475 \\
\hline 2 & 17,40 & 0,202 & 0,387 & 0,304 & 0,284 & 0,283 & 0,299 & 0.286 & 0,296 & 0,294 & 0,283 & 0,286 \\
\hline 3 & 13,00 & 0,250 & 0,249 & 0,315 & 0,335 & 0,317 & 0,320 & 0,334 & 0,334 & 0,333 & 0,335 & 0,327 \\
\hline 4 & 16,10 & 0.270 & 0,463 & 0,458 & 0,465 & 0,466 & 0,480 & 0,468 & 0,468 & 0,464 & 0,474 & 0,470 \\
\hline 5 & 16,85 & 0,280 & 0,504 & 0.515 & 0,518 & 0,520 & 0,544 & 0,523 & 0,523 & 0,519 & 0,531 & 0,528 \\
\hline 6 & 18,00 & 0,157 & 0,166 & 0,210 & 0,180 & 0,153 & 0,151 & 0,182 & 0,197 & 0,189 & 0,176 & 0,180 \\
\hline 7 & 20,40 & 0,308 & 0,723 & 0,764 & 0,731 & 0,737 & 0,759 & 0,748 & 0,750 & 0,762 & 0,763 & 0,769 \\
\hline 8 & 16,60 & 0,183 & 0,240 & 0,224 & 0,214 & 0,197 & 0,213 & 0,215 & 0,239 & 0,235 & 0,223 & 0,225 \\
\hline 9 & 15,45 & 0,204 & 0,262 & 0,266 & 0,262 & 0,253 & 0,253 & 0,262 & 0,271 & 0,270 & 0,260 & 0,259 \\
\hline 10 & 20,15 & 0,319 & 0,816 & 0,800 & 0,774 & 0,759 & 0,814 & 0,792 & 0,793 & 0,801 & 0,810 & 0,814 \\
\hline 11 & 22,80 & 0,193 & 0,347 & 0,409 & 0,327 & 0,342 & 0,291 & 0,335 & 0,348 & 0,357 & 0,329 & 0,341 \\
\hline 12 & 16,50 & 0,348 & 0,771 & 0,745 & 0,773 & 0,661 & 0,863 & 0,783 & 0,774 & 0,756 & 0,807 & 0,793 \\
\hline 13 & 21.50 & 0,310 & 0,722 & 0,823 & 0,773 & 0,787 & 0,757 & 0,795 & 0,799 & 0,819 & 0,810 & 0,820 \\
\hline 14 & 19,40 & 0,280 & 0,613 & 0,609 & 0,583 & 0,610 & 0,617 & 0,593 & 0.597 & 0,603 & 0.602 & 0,606 \\
\hline 15 & 21,30 & 0,365 & 1.133 & 1,083 & 1,050 & 0,914 & 1,188 & 1,084 & 1,085 & 1,096 & 1,116 & 1,120 \\
\hline 16 & 21,40 & 0,353 & 1,140 & 1.027 & 0,989 & 0,896 & 1,115 & 1,020 & 1,022 & 1,036 & 1,047 & 1,054 \\
\hline 17 & 18,50 & 0,212 & 0,353 & 0,356 & 0,328 & 0,339 & 0,312 & 0,332 & 0,341 & 0,342 & 0,330 & 0,334 \\
\hline 18 & 17,70 & 0,247 & 0,425 & 0,438 & 0,424 & 0,443 & 0,435 & 0,428 & 0,433 & 0,434 & 0,431 & 0,433 \\
\hline 19 & 16,95 & 0,300 & 0,668 & 0.587 & 0.594 & 0,579 & 0,637 & 0,601 & 0,599 & 0,592 & 0,613 & 0.608 \\
\hline 20 & 21,60 & 0,335 & 0.920 & 0,947 & 0,901 & 0,863 & 0,958 & 0,928 & 0,932 & 0,951 & 0.951 & 0,959 \\
\hline \multicolumn{3}{|c|}{ Total } & 11.37 & 11,36 & 10,97 & 10,61 & 11,47 & 11,18 & 11,28 & 11,33 & 11,37 & 11,4 \\
\hline \multicolumn{3}{|c|}{ Promedio } & 0,568 & 0,568 & 0,548 & 0,530 & 0,573 & 0,559 & 0,564 & 0,566 & 0,568 & 0,570 \\
\hline \multicolumn{3}{|c|}{ Desviación Estándar } & 0,287 & 0,270 & 0,264 & 0,240 & 0,305 & 0,274 & 0,270 & 0,275 & 0,285 & 0,286 \\
\hline
\end{tabular}

$\mathrm{H} \quad=$ Altura del árbol (m)

$\mathrm{D}=$ Dap del árbol

$\mathrm{V}=$ Volumen $\left(\mathrm{m}^{3}\right)$

VO $=$ Volumen observado

$\mathrm{VA1}=$ Amidon

$\mathrm{VA} 2=$ Clutter

$\mathrm{VA} 3=$ Kozak

VA4 $=$ Newnham

VA5 $=$ Rustagi $y$ Loveless

M1 = Spurr

M6 = Múltiple de tres parámetros

M7 = Schumacher $\mathrm{y}$ Hall

M8 = Spurr logarítmica

Los modelos de ahusamiento no predicen tan precisamente los volúmenes observados de los 20 árboles, como lo hacen los modelos de volumen, en contraste con los observado por Navar et al. , (1997) para Pinus hartwegii. El modelo de Amidon es el que más se aproxima, mientras que el modelo de Kozak el que menos se aproxima a los volúmenes totales observados. El modelo de Newnham sobrestima el volumen fustal total por $0,10 \mathrm{~m}^{3}$ y presenta una variación mayor en los volúmenes estimados que la mostrada por los árboles muestra. Los modelos de volumen, en especial el de Schumacher y Hall, predicen más adecuadamente los volúmenes fustales totales, promedio y sus variaciones. 


\section{Comparación entre Ecuaciones de Volumen y Ahusamiento}

Contreras (1997) reporta para la especie Pinus teocote de la Sierra Madre Occidental del estado de Durango que el modelo que mejor se ajusta a los volúmenes fustales, fuste+tocon, fuste+ramas y fuste+tocon+ramas con y sin corteza fue el de Schumacher y Hall (1933), con la siguiente ecuación:

$\mathrm{V}=\exp \left(-0,479389+2,00804 *(\mathrm{LND})+0,858299 *(\mathrm{LNH}) \operatorname{con} \mathrm{r}^{2}=0,97\right.$ y EEE $=0,1057$. El modelo de Schumacher y Hall reportado en este estudio fue el siguiente: $\mathrm{V}=\operatorname{Exp}\left(0,600417+2,008682 *(\mathrm{LND})+0,894068 *(\mathrm{LNH})\right.$, con un $\mathrm{r}^{2}=0,95$ y un $\mathrm{EEE}=0,06001$. Los modelos no presentan diferencias estadísticamente significativas en su intercepta $(\mathrm{PF}=0,55)$ ni tampoco en sus pendientes ( $\mathrm{PF}=0,647)$ (Figura 2a).

La ecuación de Spurr ha sido también reportada para Pinus teocote en la sierra Madre Occidental de Durango por Contreras (1997) como: $V=0,0501+0,3566 * D^{2} H$ $\left(\mathrm{r}^{2}=0,97\right.$ y EEE $\left.=0,106\right)$. Mientras que Jiménez (1988), determina que el mejor modelo para la especie de Pinus teocote, para otras localidades de la Sierra Madre Oriental, fue el de Spurr, con la siguiente ecuación: $V=0,008752+0,39 * D^{2} H\left(r^{2}=0,99, E E E=0,08788\right)$. El modelo ajustado de Spurr de esta investigación fue la siguiente: $\mathrm{V}=0,033415+0,370801 \mathrm{D}^{2} \mathrm{H}\left(\mathrm{r}^{2}=0,9626, \mathrm{EEE}=0,05680\right)$. El análisis de covarianza no mostró diferencias estadísticamente significativas entre las interceptas ( $\mathrm{P} F=0,43) \mathrm{ni}$ tampoco entre las pendientes ( $\mathrm{P} F=0,567)$, así como en sus parámetros y errores estándar. Las similitudes entre estos últimos modelos se pueden observar en la Figura $2 \mathrm{~b}$.

Los perfiles diámetros de los árboles muestreados en esta fracción de la Sierra Madre Oriental, trabajados por el modelo de Newnham de 16 parámetros, tienden a mostrar un menor ahusamiento en la base del fuste y una mayor cilindricidad hasta un metro antes de la porción distal del fuste en comparación con los perfiles observados por Contreras (1997) para la misma especie localizada en la Sierra Madre Occidental por el modelo de Newnham de 11 parámetros (Figura 3). Esto puede posiblemente explicarse por la menor competencia a que se encuentran sometidos los árboles estudiados para este reporte de investigación. A este respecto, Larson (1963) demostró que las diferencias del perfil diamétrico se deben parcialmente al tamaño de la copa viva que se distribuye en el fuste y el tamaño de las ramas. 


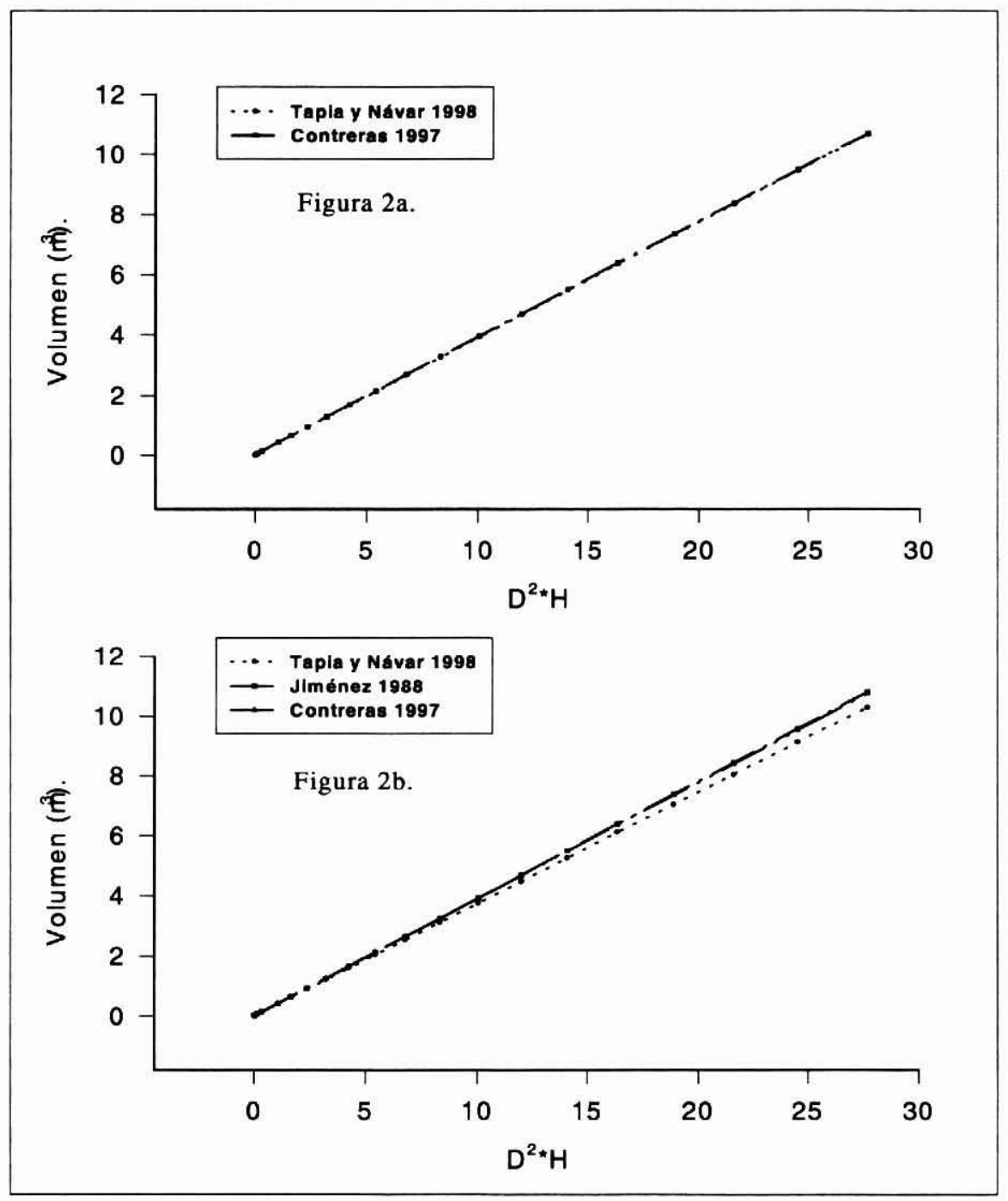

Figura 2. COMPARACIÓN DE ESTIMACIÓN DE VOLUMEN POR LA ECUACIÓN DE SPURR Y SCHUMACHER Y HALL PARA LA ESPECIE Pinus teocote DE LA SIERRA MADRE ORIENTAL Y OCCIDENTAL. 


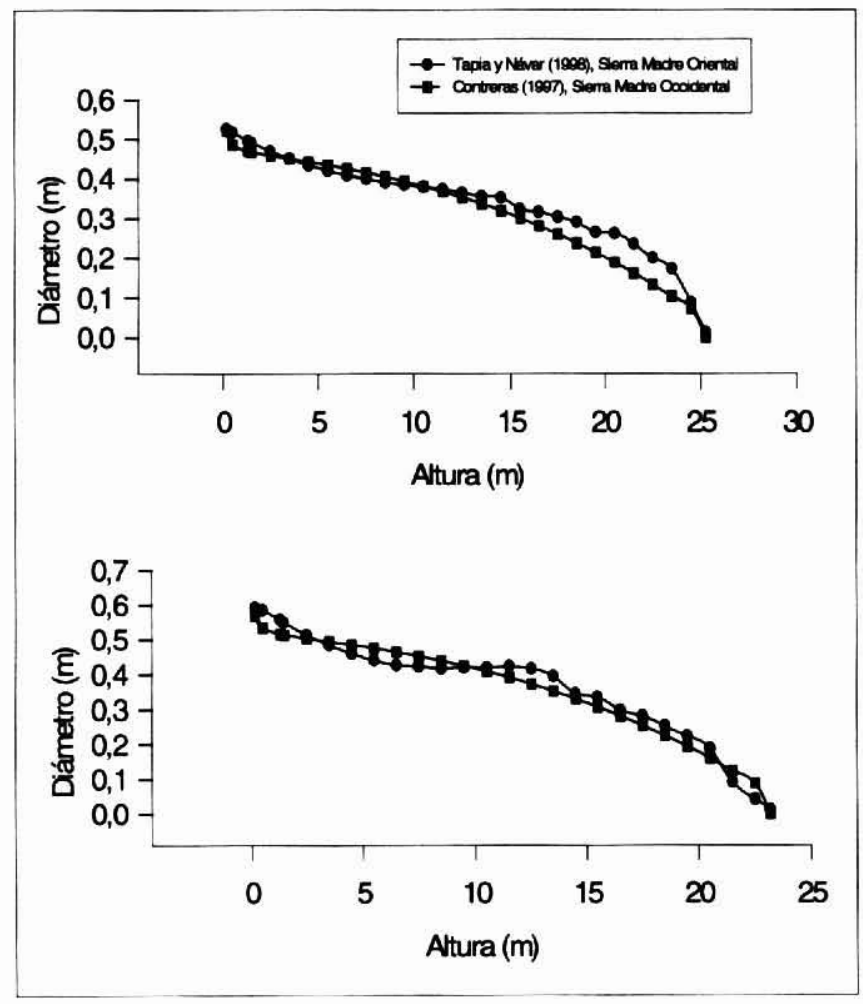

Figura 3. CURVAS DE LA ECUACIÓN DE NEWNHAM REPORTADA POR CONTRERAS (1997), EN LA SIERRA MADRE OCCIDENTAL COMPARADA CON TAPIA Y NÁVAR (1998), PARA LA SIERRA MADRE ORIENTAL.

\section{CONCLUSIONES}

Se ajustaron y probaron por bondad de ajuste 8 ecuaciones de volumen y 5 funciones de ahusamiento a 110 y 20 árboles de la especie Pinus teocote localizada en la Sierra Madre Oriental. Los modelos recomendados para estimar volúmenes fustales totales y comerciales para la especie estudiada y que pueden ser utilizados para el Norte de México son el de Schumacher y Hall principalmente, y después el de Spurr. El primero presentó parámetros similares a ecuaciones de volúmenes ajustadas para bosques de la Sierra Madre Oriental y la Sierra Madre Occidental. El segundo modelo también presenta parámetros similares, pero subestima ligeramente el volumen fustal total. La 
ecuación de ahusamiento de Newnham describe los perfiles con menor cambio de diámetro en la base del fuste, mayor cilindricidad hasta un metro de la parte distal del fuste y otro cambio drástico en diámetro en esta parte final del fuste.

\section{RECONOCIMIENTOS}

Los autores de este reporte de investigación desean hacer patente su agradecimiento al Consejo Nacional de Ciencia y Tecnología por los apoyos otorgados a través del proyecto de investigación 2452P $\mathrm{N}$ para la realización de este trabajo, al Técnico Forestal Roque Felix Cervantes Soto, a los alumnos de cuarto semestre de licenciatura Miguel Angel Aguilar Charles y Francisco Javier Sánchez Ortíz por la ayuda en el derribo y la toma de datos necesarios para esta investigación.

\section{REFERENCIAS BIBLIOGRÁFICAS}

Amidon, E. L. 1984. A general taper functional form to predic bole volume for five mixted conifer especies in California. For. Sci. 30:166-171.

Baca, H., S. Valenzuela, L. M. Torres y E. H. Cornejo. 1997. Tabla de volúmenes para regeneración de Pinus rudis en el Cerro Potosí. III Congreso Mexicano sobre Recursos Forestales. Resumen de ponencias. Nuevos paradigmas. Linares, Nuevo León. 122 p.

Clutter, J. L. 1980. Development of taper functions from variable-top merchantable volume equations. For. Sci. 26: 117-120.

De los Santos-Pozadas, H. M., J. R. Valdez-Lazalde y M. Rodriguez-Aguilar. 1995. Uso de un modelo compatible volumen-ahusamiento de Bailey en dos especies mexicanas de pinos. II Congreso de Mexicano sobre Recursos Forestales. Resumen de ponencias. Desarrollo sustentable redifiniendo el papel de los recursos forestales. Montesillo, México. 91 p.

García, E. 1985. Apuntes de climatología. U. N. A. M.

Herrera-Moncivais, Verónica. 1995. Elaboración de una tabla y tarifa de volumen para Pinus hartwgï Lindl en el Cerro Potosí, Galeana, Nuevo León. Tesis profesional. Facultad de Ciencias Forestales. Universidad Autónoma de Nuevo León. Linares, Nuevo León. 51 p.

Jiménez, J., O. Aguirre, M. Niembro, J. Návar y A. Domínguez. 1994. Determinación de la forma externa de Pinus hartwgii Lindl en el Noreste de México. Investigación Agraria Sistemas y Recursos Forestales. Ministerio de Agricultura, Pesca y Alimentación. 3 (2). España.

Jiménez Pérez, J. 1988. Elaboración de tablas y tarifas de volumen para fuste limpio de Pinus psedustrobus Lindl. y Pinus teocote Schl et Cham. en una fracción de la sierra Madre Oriental en el Noreste de México. Tesis doctoral. Facultad de Ciencias Forestales. Linares, Nuevo León. 99 p.

Jiménez Pérez, J. 1990. Aplicación de un modelo matemático para elaborar tablas y tarifas de volumen. Un ejemplo con Pinus pseudostrobus. Rep. Científico No. 16 Facultad de Ciencias Forestales. Linares, Nuevo León. 48 p.

Kozak, A. 1988. A variable-exponent taper equation. Can. J. For. Res. 18:1363-1368. 
Larson, P. R. 1963. Stem form development of forest trees. For. Sci Monograf. 5.

Márquez-Linares, M. A., J. Soto-Rodríguez y J. C. Contreras-Aviña. 1995. Tablas de volúmenes para árboles del género Pinus en el Ejido Vencedores San Dimas, Durango. II Congreso de Mexicano sobre Recursos Forestales. Resumen de ponencias. Desarrollo sustentable redifiniendo el papel de los recursos forestales. Montesillo, México. $91 \mathrm{p}$.

Martínez, M. 1948. Los pinos mexicanos. $2^{\mathrm{a}}$ ed. Editorial Botas. México. 61 p.

Návar, J., M. R. Bravo-Garza, J. Y. Uvalle-Sauceda, E. de los Ríos-Carrasco, S. M. JiménezPérez y S. A. Báez-García. 1995. Aplicación de un modelo para evaluar tarifas de volumen forestal en Pinus hartwgii en el Noreste de México. II Congreso de Mexicano sobre Recursos Forestales. Resumen de ponencias. Desarrollo sustentable redifiniendo el papel de los recursos forestales. Montesillo, México. 91 p.

Návar J., P. A. Domínguez-Calleros, J. C. Contreras-Aviña y C. Estrada M. 1997. Ajuste de siete modelos de ahusamiento a los perfiles fustales de Pinus hartwegï Lindl. Del Noreste de México. Agrociencia 31: 73-81.

Navarro, M. S. A., S. Valencia. L. M. Torres y E. H. Cornejo. 1997. Elaboración y validación de una tabla de volúmenes para Pinus cembroides en la Sierra Arteaga, Coahuila. III Congreso Mexicano sobre Recursos Forestales. Resumen de ponencias. Nuevos paradigmas. Linares, Nuevo León. 122 p.

Newnham, R.M. 1992. Variable-form taper functions for four Alberta tree species. Can. J. For. Res. 22: $210-223$.

Niembro-Barroso, M. 1992. Estimación de la forma externa del árbol mediante tres funciones de ahusamiento (Un ejemplo con Pinus hartwgii Lindl). Tesis profesional. Facultad de Ciencias Forestales. Universidad Autónoma de Nuevo León. Linares, Nuevo León. 46 p.

Rentería-Anima, J. B., H. Ramírez-Maldonado y F. J. Zamudio-Sánchez. 1995. Estimación de volumen comercial de Pinus cooperi blanco mediante modelos de ahusamiento en Durango. II Congreso de Mexicano sobre Recursos Forestales. Resumen de ponencias. Desarrollo sustentable redifiniendo el papel de los recursos forestales. Montesillo, México. 91 p.

Rustagi, K. P. and Loveless. R. S., 1991. Compatible variable-form volume and stem profile equations for Douglas-fir. Can. J. For. Res. 21:143-151.

Schumacher, F. X.. and F. D. S. Hall. 1933. Logarihmic expression of timber-tree volume. J. Agr. Res. 47:719734.

Secretaría de Agricultura y Recursos Hidráulicos. 1994. Inventario nacional forestal periódico. Memoria nacional. Subsecretaría forestal y de fauna silvestre. México, D. F. 81 p.

Spurr, S. H. 1952. Forest Inventory. The Ronald Press Company. New York. 476 p.

Tapia, J. y J. Návar. 1997. Observaciones preliminares sobre la determinación del número de datos mínimos para la estimación de funciones de ahusamiento y volumen para Pinus durangensis y Pinus teocote. III Congreso Mexicano sobre Recursos Forestales. Resumen de ponencias. Nuevos paradigmas. Linares, Nuevo León. 122 p. 
Torres-Rojo, J. M., S. O. Magaña T. y G. A. Valles G. 1993. Funciones de ahusamiento para 8 especies Forestales del Estado de México. I Congreso Mexicano de Recursos Forestales, Resumen de Ponencias. Saltillo, Coahuila. 109 p.

Zepeda-Bautista, M. y A. Domínguez-Pereda. 1997. Ecuaciones de ahusamiento para tres especies de pino, del éjido "El largo", Chihuahua. III Congreso Mexicano sobre Recursos Forestales. Resumen de ponencias. Nuevos paradigmas. Linares, Nuevo León. 122 p. 